\title{
Superior radiation tolerant materials: Amorphous silicon oxycarbide
}

Michael Nastasi ${ }^{1,2^{*}}$, Qing $\mathrm{Su}^{1}$, Lloyd Price ${ }^{3}$, Juan A. Colón Santana ${ }^{4}$, Tianyi Chen ${ }^{3}$, Robert Balerio $^{3}$, and Lin Shao ${ }^{3}$

${ }^{1}$ Nebraska Center for Energy Sciences Research, University of Nebraska-Lincoln, Lincoln, NE 68583-0857, USA

${ }^{2}$ Department of Mechanical and Materials Engineering, University of Nebraska-Lincoln, Lincoln, NE 68583-0857, USA

${ }^{3}$ Department of Nuclear Engineering, Texas A\&M University, College Station, TX 77843-3128, USA

${ }^{4}$ Department of Physics, Northern Illinois University, DeKalb, IL 60115, USA

\begin{abstract}
We studied the radiation tolerance of amorphous silicon oxycarbide (SiOC) alloys by combining ion irradiation, X-ray diffraction (XRD) and transmission electron microscopy (TEM). The amorphous $\mathrm{SiOC}$ alloys thin films were grown via co-sputtering from $\mathrm{SiO}_{2}$ and $\mathrm{SiC}$ (amorphous phase) targets either on a surface oxidized Si (100) substrate or on a sodium chloride substrate. By controlling the sputtering rate of each target, SiOC alloys with different compositions (1:2, 1:1, 2:1 ratios) were obtained. These alloys were irradiated by $100 \mathrm{keV} \mathrm{He}^{+}$ ions at both room temperature and $600{ }^{\circ} \mathrm{C}$ with damage levels ranging from 1 to 20 displacements per atom (dpa). TEM characterization shows no sign of crystallization, void formation or segregation in all irradiated samples. Our findings suggest that SiOC alloys are a class of promising radiation-tolerant materials.
\end{abstract}

Keyword: Radiation damage, radiation tolerant materials, amorphous, Silicon oxycarbide

*Corresponding author. Tel.: +1 (402) 472-3852

Email address: mnastasi2@unl.edu (Michael Nastasi) 


\section{Introduction}

To extend the lifetime of current nuclear power plants and to meet the needs of advanced reactor designs, it is necessary to develop radiation tolerant alloys without significant structural changes and serious thermal/mechanical degradation under harsh environments [1-5]. Different strategies have been explored to improve radiation tolerance of structural materials. In particular, efforts have been made to introduce interfaces as point-defect sinks to remove radiation damage and suppress swelling. For example, oxide dispersion strengthened (ODS) steels with a large amount of interfaces between the matrix and nanoscale oxide precipitates have proven to have good swelling resistance and good creep resistance [6,7]. Nanoscale metallic interfaces, e.g. interfaces of face-centered cubic and body-centered cubic alloys, have displayed strong sink strength and suppressed He bubble formation [8, 9]. Nanocrystalline metals, although facing challenges of grain stability under irradiation, have shown enhanced radiation resistance due to grain boundary assisted defect annihilation $[10,11]$.

Unlike crystalline solids, whose radiation responses have been studied for decades [12, 13], radiation effects in amorphous alloys have received much less attention [14] and were therefore still largely unknown. Molecular dynamics (MD) simulations of damage cascade development in ion irradiated amorphous materials did not reveal partial crystallization. Instead, radiation was found to create excessive free volume in localized region and these "defects" can anneal out on a time scale of about 100ps at room temperature, and thus sustaining their glassy states[15]. Because amorphous materials possess no long range order, they do not contain conventional crystal defects such as vacancies, interstitials, or dislocations [16]. Therefore they offer the possibility of eliminating the root cause responsible for radiation damage in crystalline solids - namely the production of point defects and defect clusters - and may serve as the basis 
for developing a new class of radiation tolerant structural materials. Fig. 1 shows two possible consequences of amorphous alloy structural evolutions after radiation. One is irradiation-induced crystallization. Since collision cascades caused by ion bombardments will lead to high density atomic displacements and possibly correlated atomic re-arrangements, the nucleation of a crystalline phase may be possible under irradiation [17]. The second possible consequence of amorphous alloys under irradiation is that they maintain their amorphous structure. Although glassy states are generally metastable, some glasses may possess large kinetic barriers to the nucleation of a crystalline phase. Several studies on metallic glasses have shown that a direct crystallization in the damage cascade core is unlikely considering because the cooling rates during the thermal spike quenching stages are typically a few orders of magnitudes higher than the critical cooling rates required for crystallization [18]. Therefore, the local molten zone formed in the damage cascade core maintains a glassy state upon thermal spike quenching and subsequent cooling at longer time scales.

In the present study, silicon oxycarbide (SiOC) was selected due to several reasons. First, this class of amorphous ceramics has been shown to have crystallization temperatures in excess of $1300{ }^{\circ} \mathrm{C}$ and good oxidation and creep resistance [19-22]. Second, it is convenient to fabricate amorphous SiOC alloy thin films with controlled composition by the magnetron sputtering method [23]. The high crystallization temperature indicates that the amorphous state is highly stable. In addition, good mechanical properties make it a promising candidate for a structural material. Third, the irradiation resistance of an amorphous alloy is believed to be closely coupled to its crystallization temperature. Therefore, $\mathrm{SiOC}$ has an extremely high crystallization temperature which suggests that service operating temperatures of this material should be well above $500{ }^{\circ} \mathrm{C}$. 


\section{Experimental}

There were three types of SiOC film systems used in the present study. The first type SiOC films was deposited on a sodium chloride substrate. After dissolving the substrate in deionized water, SiOC films were self-suspended on TEM grids. These TEM specimens were subject to a series of repeated He ion irradiations followed by TEM characterization after each He ion fluence. Such self-supported membrane films, however, were not suitable for irradiation at elevated temperatures. To alleviate this issues, the second type of SiOC films were deposited on surface oxidize $\mathrm{Si}$ substrates with their top $\mathrm{SiO}_{2}$ layer thickness of $300 \mathrm{~nm}$. After ion irradiation at elevated temperature, either a focused ion beam technique or a conventional dimple and grinding followed by ion-milling technique were used to prepare specimens for cross-section TEM characterization. The third type of SiOC films were deposited on carbon substrates. The low Z substrate allows for Rutherford backscattering spectrometry (RBS) composition analysis of the deposited SiOC films.

Amorphous SiOC films were grown via radio frequency (RF) co-sputtering from $\mathrm{SiO}_{2}$ and $\mathrm{SiC}$ targets at room temperature. Three different compositions of SiOC alloys were obtained by varying the sputtering rates of the $\mathrm{SiO}_{2}$ and $\mathrm{SiC}$ targets. The $\mathrm{SiO}_{2}$ to $\mathrm{SiC}$ sputtering ratios in three alloyswere 1:1, 1:2 and 2:1. Both the $\mathrm{SiC}$ (purity 99.5\%) and $\mathrm{SiO}_{2}$ (purity 99.995\%) targets were purchased from AJA International, Inc. The deposited SiOC film thickness was 300 nm. The base pressure prior to depositions was $9.8 \times 10^{-6} \mathrm{~Pa}$ and the Ar partial pressure during deposition was $\sim 0.65 \mathrm{~Pa}$.

RBS analysis of SiOC films deposited on carbon substrates was performed by using 2 $\mathrm{MeV} \mathrm{He}$ ions. A semiconductor detector with a $20 \mathrm{keV}$ energy resolution was mounted at a 160 
degree backscattering angle for spectra collection. For ion irradiation, the SiOC films were irradiated at room temperature and $600{ }^{\circ} \mathrm{C}$ using $100 \mathrm{keV} \mathrm{He}$ ions. The $\mathrm{He}^{+}$irradiation damage events in SiOC films were obtained by the Stopping and Range of Ions in Matter (SRIM) simulation [24]. The corresponding dpa of SiOC films was calculated based on SRIM simulation. The temperature during ion irradiation was automatically controlled based on temperature readings from a thermal couple attached to the target holder. The base pressure during irradiation

was better than $5 \times 10^{-5}$ torr. The crystallinity of the SiOC films was examined using X-ray Diffraction (XRD) by a PANalytical XRD Analyser with incident X-rays from $\mathrm{Cu} \mathrm{K} \alpha$ (wavelength of $1.5406 \AA$ A). A FEI Tecnai G2 F20 Transmission Electron Microscope (TEM) was employed to investigate the microstructure of the SiOC thin films before and after irradiation. The typical operation voltage for TEM is $200 \mathrm{kV}$.

\section{Results}

Figure 2 shows a typical RBS spectrum obtained from the 1:2 SiOC film deposited on a carbon substrate. The solid lines are fitting from RUMP simulations[25]. RBS analysis reveals the following nominal compositions (atom \%): Alloy 1:1 Si-30\%, O-40\%, C-30\%; Alloy 2:1 Si$30 \%, \mathrm{O}-53 \%, \mathrm{C}-17 \%$, and Alloy 1:2 Si-33\%, O-35\%, C-32\%. Repeated characterizations on several different positions show a high uniformity of film compositions.

We selected $100 \mathrm{keV} \mathrm{He}$ ion irradiation due to the needs to maximize displacement efficiency and minimize He doping in the SiOC layer. Fig. 3 shows the depth profiles of implanted He atoms, obtained by the Stopping and Range of Ions in Matter (SRIM) simulation [24]. The majority of He atoms come to rest in either the $\mathrm{Si}$ substrate or the thermalize $\mathrm{SiO}_{2}$ layer, with almost a negligible amount of $\mathrm{He}$ in the $\mathrm{SiOC}$ layer. Fig. 4a-e show high resolution 
TEM micrographs obtained from self-suspended SiOC 1:1 films before and after irradiation to $1.2,6,12$, and $18 \mathrm{dpa}$ at room temperature, respectively. The as-prepared film shows no sign of long range orders and the glassy state is confirmed. No crystallization or segregation are observed, up to the highest radiation damage level (18 dpa). The inset of each figure is corresponding Fast Fourier Transform (FFT) image; the diffuse ring confirms that the SiOC thin films maintain their amorphous state after irradiation. These data clearly demonstrate the superior room temperature irradiation tolerance of the SiOC.

To further investigate structural stability of SiOC films at elevated temperature, $\mathrm{He}^{+}$ irradiations were conducted for SiOC thin films $(1: 2,1: 1,2: 1$ ratios $)$ at $600{ }^{\circ} \mathrm{C}$. These films are deposited on surface oxidized Si substrates. Fig. 4a, 4b, and 4c are XRD patterns of SiOC films of compositions 1:2, 1:1 and 2:1, respectively. For each composition, comparisons were made for 0,5 , and 10 dpa irradiation. For all spectra, only silicon substrate signals corresponding to (200) and (400) diffraction peaks were observed. No other peaks are observed in the spectra, suggesting the absence of SiOC crystallization. Fig. 6a shows a cross-section TEM micrograph of $\mathrm{SiOC} 1: 2$ film irradiated to $10 \mathrm{dpa}$ at $600^{\circ} \mathrm{C}$. The layered structures for $\mathrm{SiOC}$ film, $\mathrm{SiO}_{2}$, and $\mathrm{Si}$ substrate were clearly visible. In $\mathrm{Si}$, and within $200 \mathrm{~nm}$ distance away from the $\mathrm{SiO}_{2} / \mathrm{Si}$ interface, there was a heavily damaged layer, featured with high density dislocations and defect clusters. The location of the damage layer agrees with the SRIM prediction. Different from Si substrate, there were no structural changes observed from the top SiOC film. Fig. 6b shows high resolution TEM image of the top SiOC film. No long range order or nanocrystals are observed. The selected area diffraction analysis further confirms their glassy state.

The high resolution cross-section TEM images from SiOC thin films (1:2, 1:1, 2:1 ratios) after 20 dpa irradiation at $600{ }^{\circ} \mathrm{C}$ are shown in Fig. 7a, 7b and 7c, respectively. All films retain 
their amorphous structure without crystallization or void formation, as confirmed by the diffusive halo ring features in each corresponding select-area diffraction pattern. For the SiOC 1:2 sample, a few tiny black dots are observed in the bright-field TEM image. We cannot exclude the possibility of phase segregation but it is most likely caused by contamination from FIB process. Energy-dispersive X-ray spectroscopy (EDS) was performed on the black dots and found heavier elements including $\mathrm{Fe}, \mathrm{Cu}$, etc. All these observations suggest that the SiOC films are stable up to $20 \mathrm{dpa}$ irradiation at $600^{\circ} \mathrm{C}$.

\section{Discussion}

\subsection{Composition of SiOC films}

Silicon oxycarbide is a class of non-crystalline and disordered ceramic materials which is usually synthesized by chemical methods including sol-gel[26], chemical vapor deposition[27], etc. To minimize the chemical residue and formation of free carbon, RF-magnetron co-sputtering of $\mathrm{SiC}$ and $\mathrm{SiO}_{2}$ targets without direct heating of the substrate was used in the present study. The defining structural unit of SiOC alloy is denoted by $\left(\mathrm{SiO}_{\mathrm{x}} \mathrm{C}_{4-\mathrm{x}}\right)$ with $\mathrm{x}=1,2$, or 3 , where silicon atoms are bonded to both carbon and oxygen atoms in a tetrahedral formation. Those randomly distributed and oriented $\mathrm{SiO}_{\mathrm{x}} \mathrm{C}_{4-\mathrm{x}}$ units are what comprise amorphous SiOC films. Different compositions of SiOC thin films result in different combinations and distributions of $\mathrm{SiOC}_{3}$, $\mathrm{SiO}_{2} \mathrm{C}_{2}$ and $\mathrm{SiO}_{3} \mathrm{C}$. There are no thermodynamically stable silicon oxycarbide crystalline phases found naturally, which indicates a good thermal stability of amorphous SiOC alloys [28, 29].

4.2 Irradiation-induced crystal-to-amorphous transition versus amorphous-to-crystal transition 
Amorphization is one of the extreme consequences of ion-beam irradiation of crystalline materials[30] and it has been demonstrated that several crystalline solid systems undergo a crystal-to-amorphous transition under irradiation[31, 32]. Irradiation-induced amorphization results from two processes: creation of disorder or defect in crystalline solids by ion beam irradiation and preservation of defects due to constrained atomic or molecular movement. The typical example is temperature-dependent amorphization of SiC using different ion species [33]. There are several findings in this system: (1) at the same temperature, the heavier the ion is, the lower the threshold dose for amorphization is. (2) For the same ion species, the threshold dose for amorphization increases as irradiation temperature increases. (3) Above a critical temperature, the threshold dose approaches infinity and $\mathrm{SiC}$ cannot be amorphized. Therefore, there is a temperature limit to avoid a crystal-to-amorphous transition for $\mathrm{SiOC}$ regardless of the bombarding ion species.

\subsection{Thermodynamic after irradiation}

Unlike the creation of vacancies and interstitials in solid crystalline systems during irradiation, free volume is generated during irradiation in amorphous alloys. One key question to be answered is whether crystallization takes place in amorphous alloys along the ion track. This depends on the relative rates of two processes. One is the quenching rate of the thermal spike associated with damage cascade creation and diffusion of excessive free volume created along the ion track. The other is the relaxation time required for crystallization. Based on the results of the room temperature radiation experiment, there is no crystal formation after a damage level of $18 \mathrm{dpa}$ is reached. It suggests that the quenching rate is faster than the relaxation time of SiOC crystallization. In addition, crystallization of ceramic materials, such as $\mathrm{SiOC}$, requires a high 
degree of correlated atomic movements due to its complicated directional bonding. Therefore, it is unlikely to occur in amorphous SiOC alloys under RT irradiation. The expected consequence of $\mathrm{RT}$ radiation is a local $\mathrm{SiO}_{\mathrm{x}} \mathrm{C}_{4-\mathrm{x}}$ unit rearrangement, which is difficult to induce microstructure changes at large spatial scales.

However, if the substrate is heated at an elevated temperature during irradiation, the quenching rate can be significantly reduced to allow possible crystallization along the ion tracks. It is very important to observe that $\mathrm{SiOC}$ alloy thin films still remain amorphous up to 20 dpa at $600{ }^{\circ} \mathrm{C}$. Also, all SiOC alloys $(1: 1,2: 1$ and 1:2) retain an amorphous structure after radiation, indicating that within a certain envelope of irradiation conditions and composition range, this class of SiOC alloys has a high level of radiation stability. Further studies with higher dpa values and higher temperatures are currently undertaken. The findings from the present work suggest a superior stability under even harsher environments.

\section{Conclusions}

We found that SiOC thin films remain amorphous after irradiation up to $18 \mathrm{dpa}$ at room temperature and up to $20 \mathrm{dpa}$ at $600^{\circ} \mathrm{C}$. The observations suggest that atomic displacing processes and subsequent structural changes do not lead to crystallization and segregation. The study demonstrated a very promising class of materials with superior radiation tolerance properties.

\section{Acknowledgements}

We acknowledge financial support from the DoE Office of Nuclear Energy, Nuclear Energy Enabling Technologies, award DE-NE0000533. The work was carried out in part in the 
Central Facilities of the Nebraska Center for Materials and Nanosicence, which is supported by the Nebraska Research Initiative.

\section{References}

[1] G.R. Odette, M.J. Alinger, B.D. Wirth. Annual Review of Materials Research 38 (2008) 471-503.

[2] E.E. Bloom, J.T. Busby, C.E. Duty, P.J. Maziasz, T.E. McGreevy, B.E. Nelson, B.A. Pint, P.F. Tortorelli, S.J. Zinkle. Journal of Nuclear Materials 367 (2007) 1-10.

[3] E.E. Bloom. Journal of Nuclear Materials 258-263, Part 1 (1998) 7-17.

[4] T. Muroga, M. Gasparotto, S.J. Zinkle. Fusion Engineering and Design 61-62 (2002) 13-25.

[5] G.S. Was. Journal of Nuclear Materials 367-370, Part A (2007) 11-20.

[6] G.R. Odette, D.T. Hoelzer. Jom 62 (2010) 84-92.

[7] C.P.C. Wong, R.E. Nygren, C.B. Baxi, P. Fogarty, N. Ghoniem, H. Khater, K. McCarthy, B. Merrill, B. Nelson, E.E. Reis, S. Sharafat, R. Schleicher, D.K. Sze, M. Ulrickson, S. Willms, M. Youssef, S. Zinkle, A. Team. Fusion Engineering and Design 49-50 (2000) 709-717.

[8] A. Misra, M.J. Demkowicz, X. Zhang, R.G. Hoagland. Jom 59 (2007) 62-65.

[9] A. Kashinath, A. Misra, M.J. Demkowicz. Physical Review Letters 110 (2013).

[10] X.M. Bai, A.F. Voter, R.G. Hoagland, M. Nastasi, B.P. Uberuaga. Science 327 (2010) 1631-1634.

[11] M. Samaras, P.M. Derlet, H. Van Swygenhoven, M. Victoria. Physical Review Letters 88 (2002).

[12] W.J. Weber, R.C. Ewing, L.M. Wang. Journal of Materials Research 9 (1994) 688-698.

[13] R. Sizmann. Journal of Nuclear Materials 69-70 (1978) 386-412.

[14] R.S. Averback. Mrs Bulletin 16 (1991) 47-52.

[15] M.J. Demkowicz. unpublished.

[16] P. Chaudhari, F. Spaepen, P.J. Steinhardt. Topics in Applied Physics 53 (1983) 127-168.

[17] Y. Chen, L. Jiao, C. Sun, M. Song, K.Y. Yu, Y. Liu, M. Kirk, M. Li, H. Wang, X. Zhang. Journal of Nuclear Materials 452 (2014) 321-327.

[18] J. Carter, E.G. Fu, M. Martin, G. Xie, X. Zhang, Y.Q. Wang, D. Wijesundera, X.M. Wang, W.-K. Chu, L. Shao. Scripta Materialia 61 (2009) 265-268.

[19] G.D. Soraru, D. Suttor. Journal of Sol-Gel Science and Technology 14 (1999) 69-74.

[20] G.D. Soraru, E. Dallapiccola, G. DAndrea. Journal of the American Ceramic Society 79 (1996) 2074-2080.

[21] R. Harshe, C. Balan, R. Riedel. Journal of the European Ceramic Society 24 (2004) 3471-3482.

[22] T. Rouxel, G. Massouras, G.D. Soraru. Journal of Sol-Gel Science and Technology 14 (1999) 87-94.

[23] J.V. Ryan, C.G. Pantano. Journal of Vacuum Science \& Technology A 25 (2007) 153-159. 
[24] J.B.B. J.F. Ziegler, U. Littmark. The Stopping and Range of lons in Solids, Pergamon Press, New York (1985).

[25] L.R. Doolittle. Nuclear Instruments and Methods in Physics Research Section B: Beam Interactions with Materials and Atoms 9 (1985) 344-351.

[26] G.D. Sorarù, G. D'Andrea, R. Campostrini, F. Babonneau, G. Mariotto. Journal of the American Ceramic Society 78 (1995) 379-387.

[27] S. Gallis, M.B. Huang, H. Efstathiadis, E. Eisenbraun, A.E. Kaloyeros, E.E. Nyein, U. Hommerich. Applied Physics Letters 87 (2005).

[28] L. Bois, J. Maquet, F. Babonneau, D. Bahloul. Chemistry of Materials 7 (1995) 975-981.

[29] H. Zhang, C.G. Pantano. Journal of the American Ceramic Society 73 (1990) 958-963.

[30] L.W. Hobbs, F.W. Clinard, S.J. Zinkle, R.C. Ewing. Journal of Nuclear Materials 216 (1994) 291-

321.

[31] H.M. Naguib, R. Kelly. Radiation Effects and Defects in Solids 25 (1975) 1-12.

[32] B.X. Liu, W.S. Lai, Q. Zhang. Materials Science and Engineering: R: Reports 29 (2000) 1-48.

[33] L.L. Snead, S.J. Zinkle, J.C. Hay, M.C. Osborne. Nuclear Instruments \& Methods in Physics Research Section B-Beam Interactions with Materials and Atoms 141 (1998) 123-132. 
Figures and Figure captions

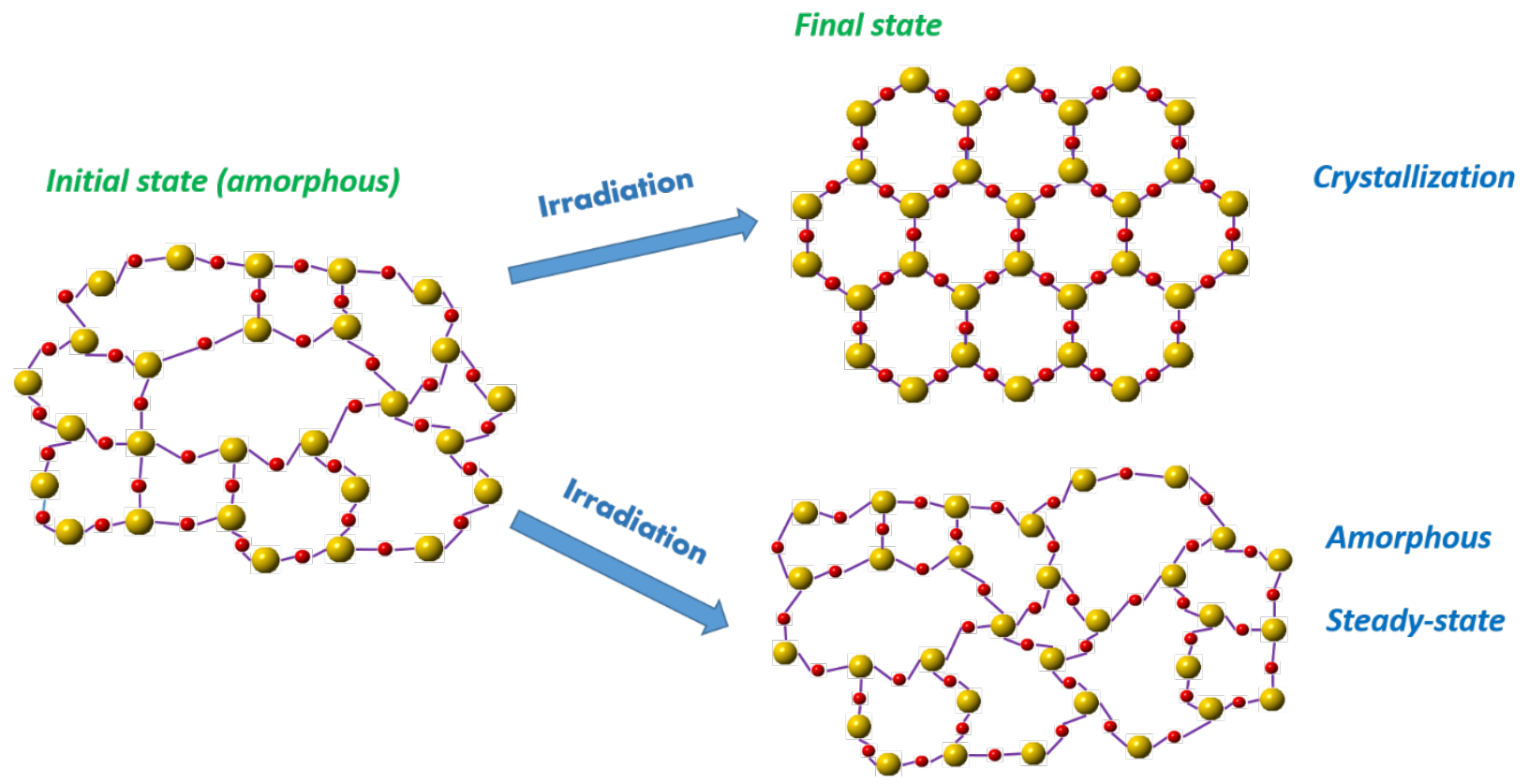

Fig. 1. The schematic illustration of two possible irradiation-induced structure for initial amorphous material: one is to become crystalline and the other is to remain amorphous. 


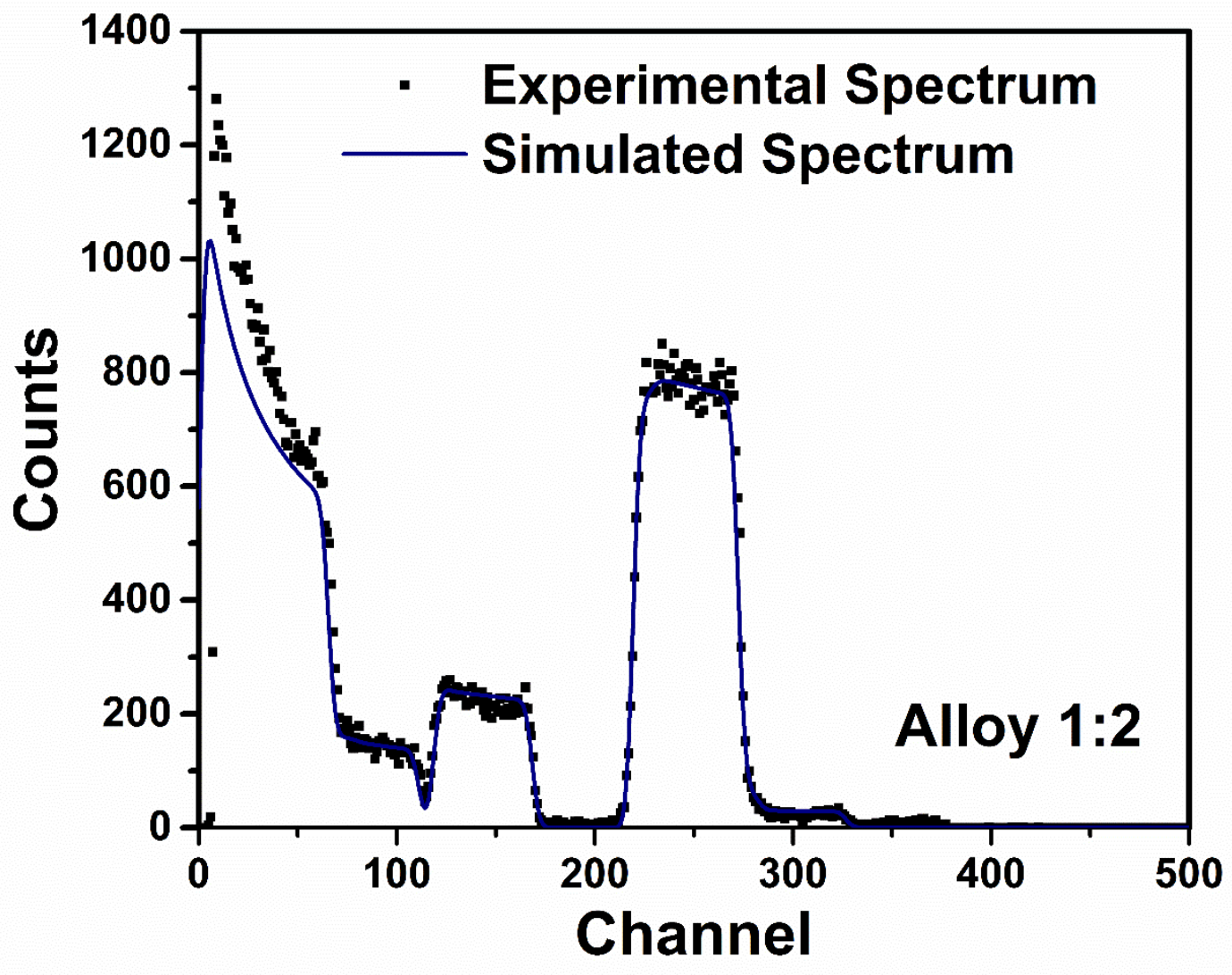

Fig. 2. The typical experimental (scattered point) and simulated (solid line) RBS spectrum for SiOC alloy (1:2). The composition of SiOC alloy is obtained by properly fitting of experimental RBS data. 


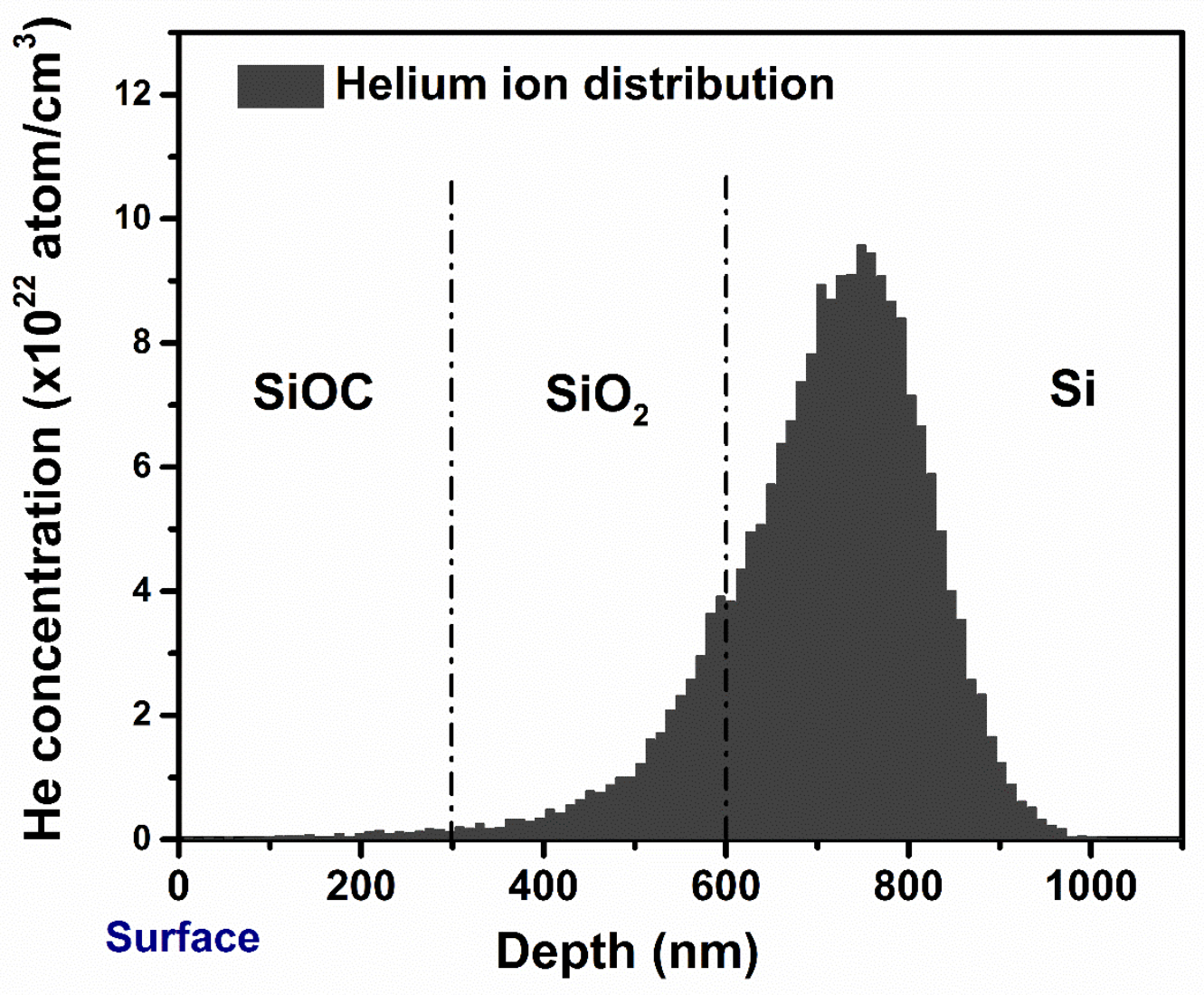

Fig. 3. The helium concentration as function of penetrating depth simulated by TRIM ( 20 dpa, dose of $2.3 \mathrm{E} 18 \mathrm{ion} / \mathrm{cm}^{2}$ ). According to the simulation, most of helium ions penetrate through the $\mathrm{SiOC}$ layer and rest in $\mathrm{Si}$ and $\mathrm{SiO}_{2}$ layer. It results in pure atomic displacement in $\mathrm{SiOC}$ layer. 


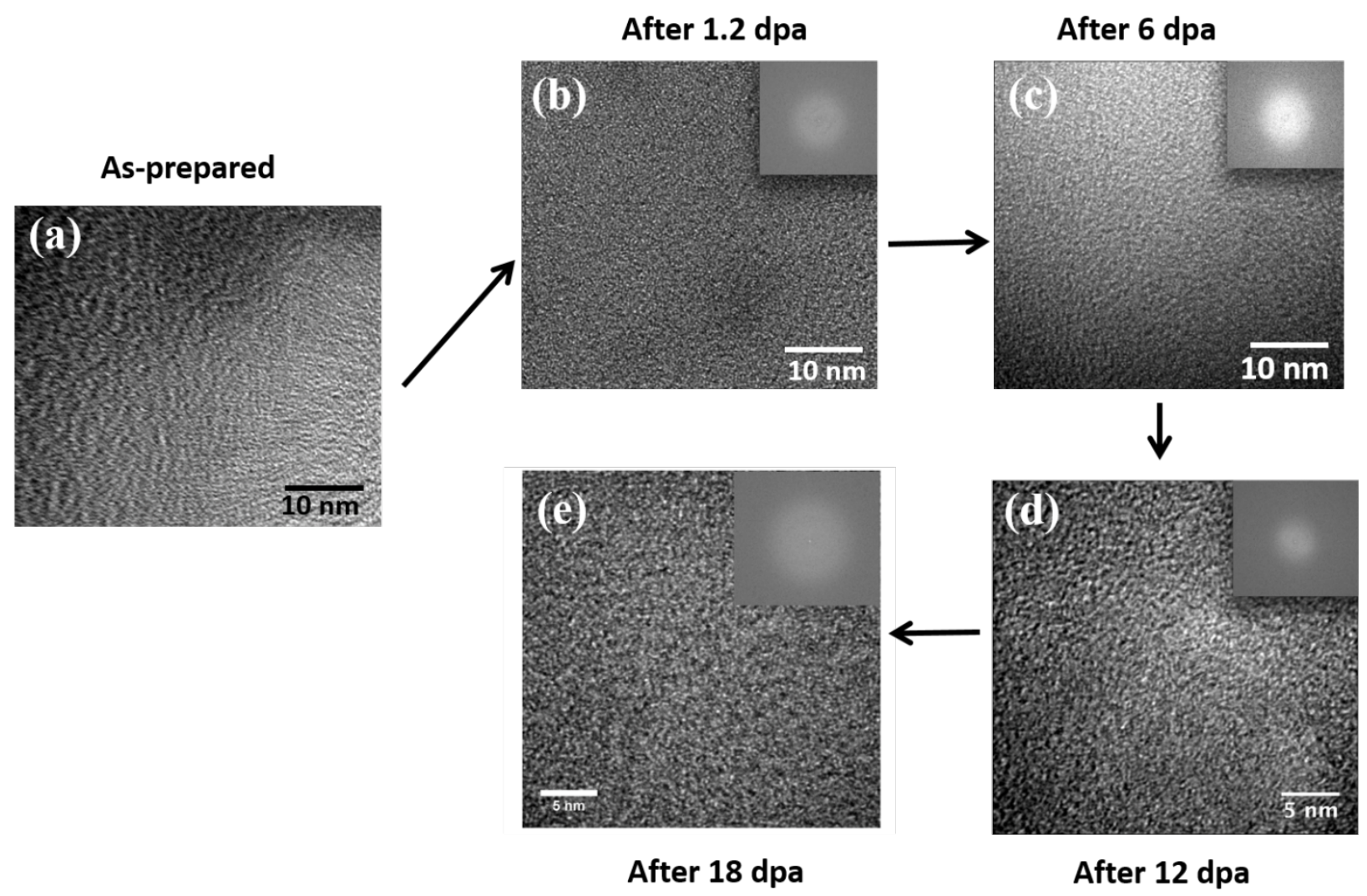

Fig. 4. The plan-view TEM image of SiOC (1:1) thin film irradiated at different levels (a) asprepared film, (b) $1.2 \mathrm{dpa}$, (c) $6 \mathrm{dpa}$, (d) $12 \mathrm{dpa}$, (e) $18 \mathrm{dpa}$ at room temperature. No observed structure change suggests superior room temperature radiation tolerance property for SiOC thin films. 

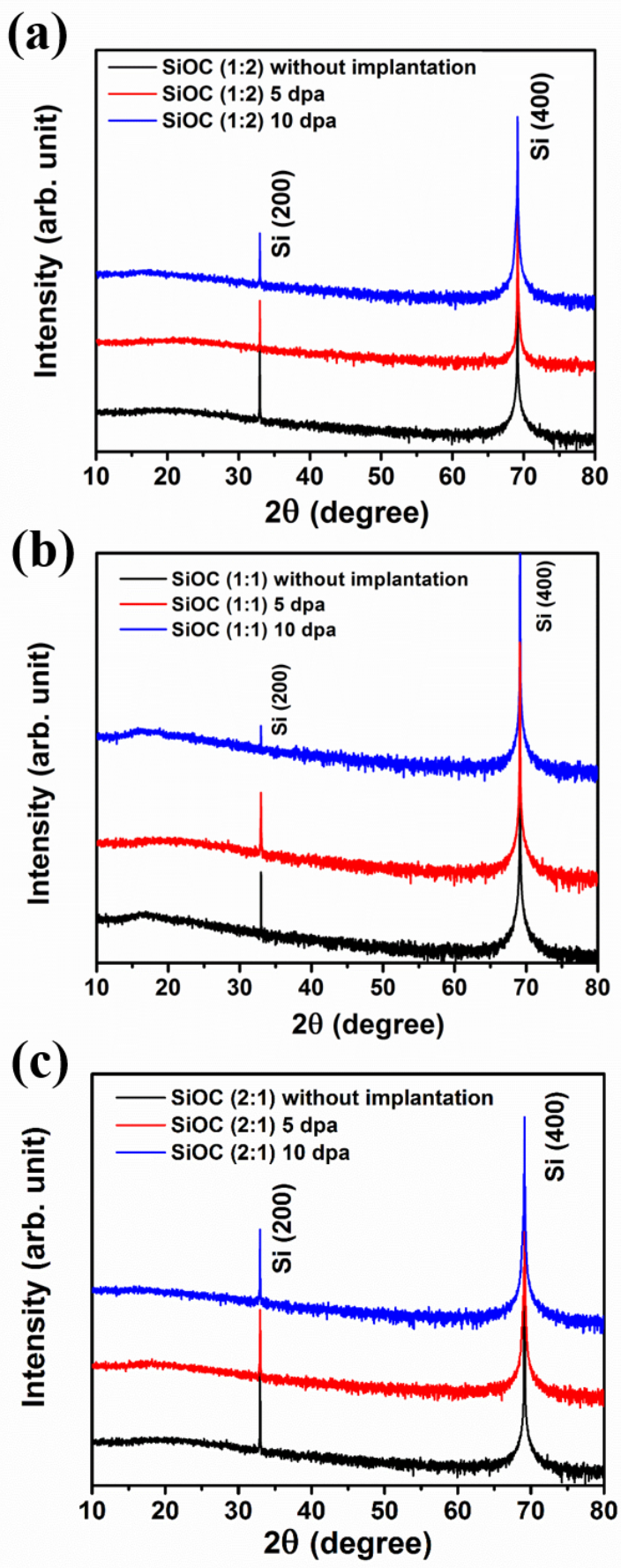

Fig. 5. The XRD of SiOC alloys thin films with different compositions (a) 1:2, (b) 1:1 and (c) 2:1 before and after irradiation at $600{ }^{\circ} \mathrm{C}$. There is no observed XRD pattern difference for SiOC films after irradiation by $10 \mathrm{dpa}$ at $600{ }^{\circ} \mathrm{C}$. The results suggest no crystal phase formation for SiOC films in a macroscopic scale after irradiation. 

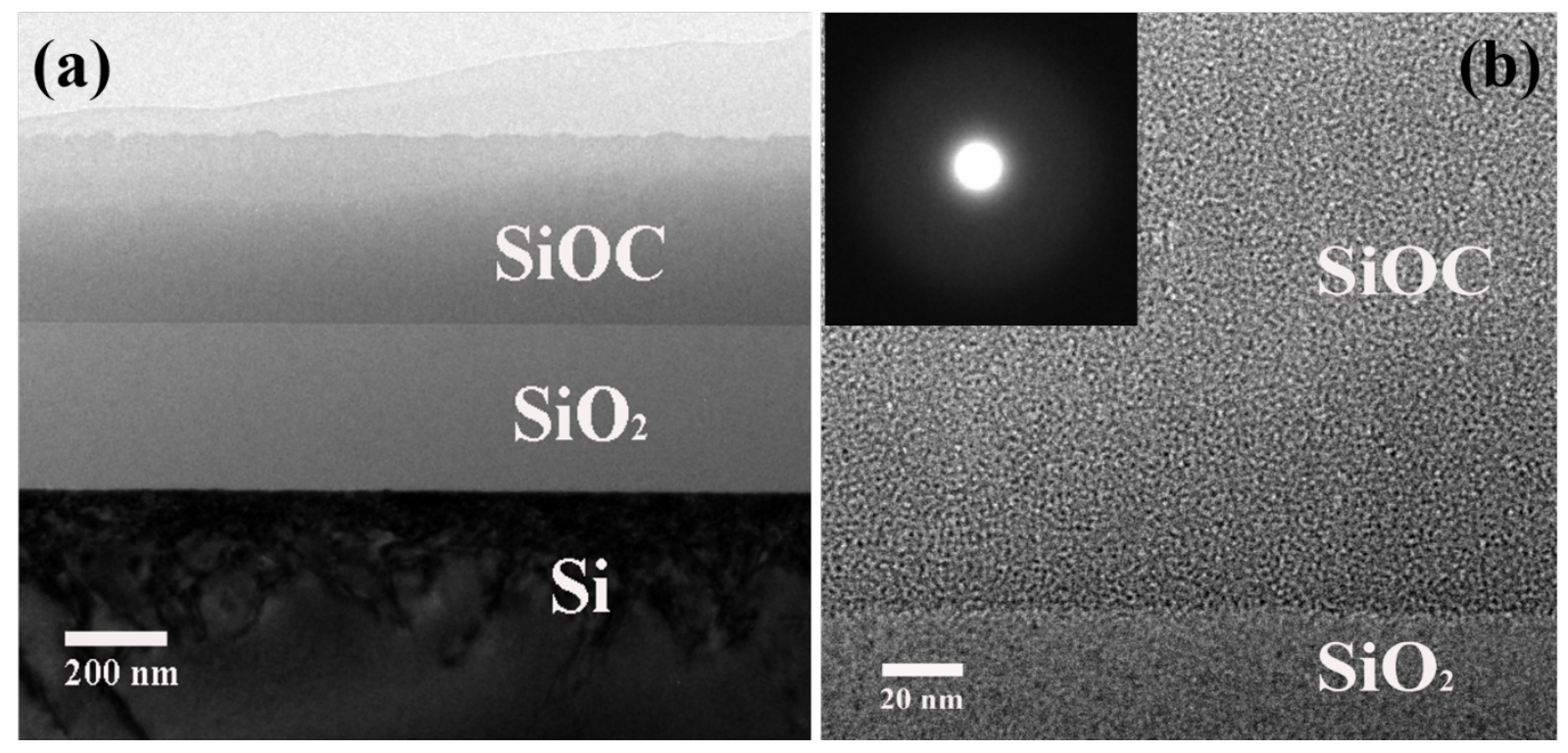

Fig. 6. (a) Typical low magnification cross-section TEM image of SiOC thin film after irradiation by $10 \mathrm{dpa}$ at $600{ }^{\circ} \mathrm{C}$. (b) Magnified cross-section TEM image of SiOC thin film exhibiting amorphous structure after irradiation at $600{ }^{\circ} \mathrm{C}$. The inset is the corresponding diffraction pattern demonstrating that the film still remain amorphous after irradiation. 

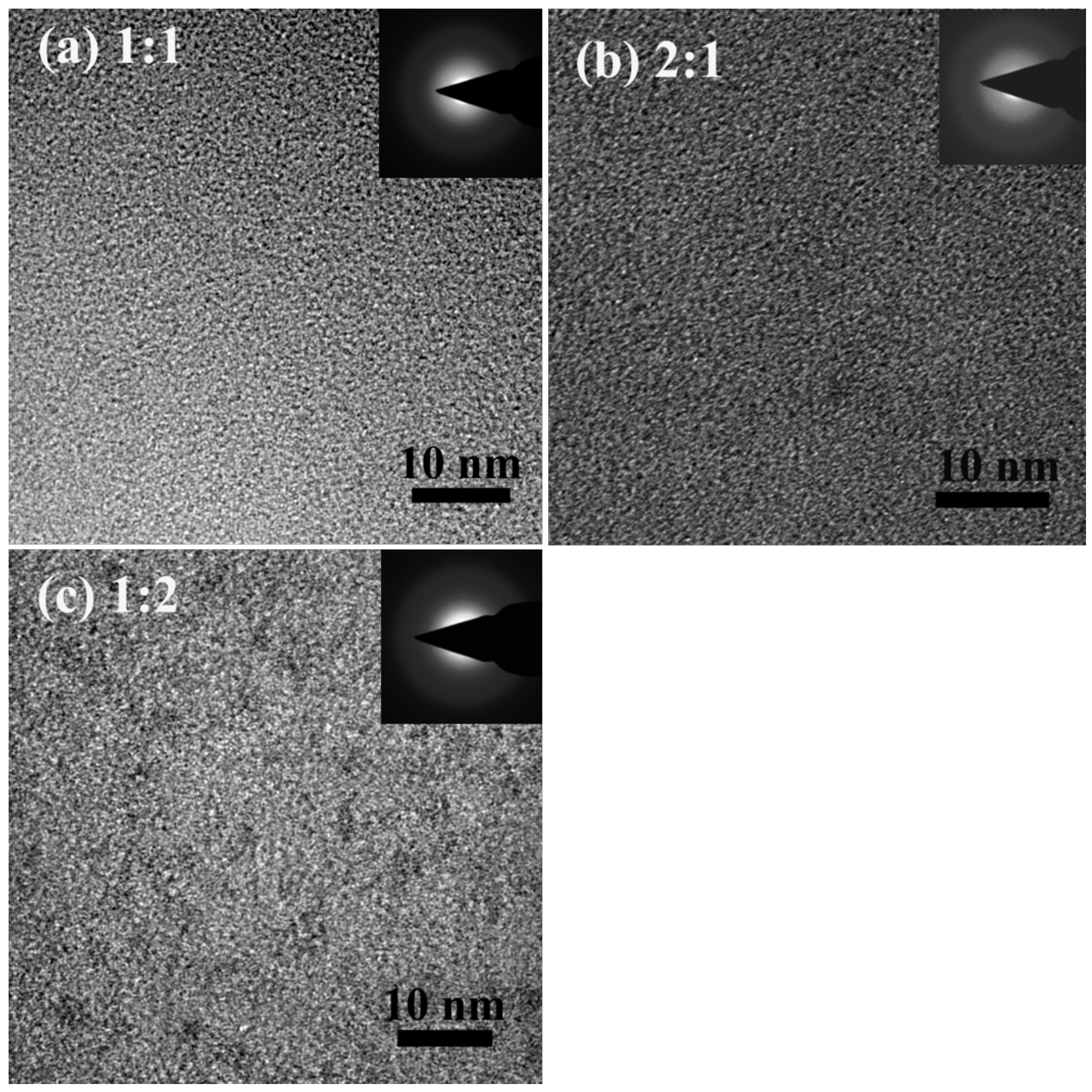

Fig. 7. The cross-section TEM images of SiOC thin films with different compositions (a) 1:1, (b)

2:1 and (c) 1:2 after irradiation by $20 \mathrm{dpa}$ at $600^{\circ} \mathrm{C}$. All of the films exhibit amorphous structure after irradiation. The inset of each figure is the corresponding diffraction pattern. The diffuse diffraction ring suggests no crystal formation for $\mathrm{SiOC}$ thin films after hot irradiation. 